Acta vet. scand. 1960, 1, 281-293.

Aus dem Veterinärmedizinischen Staatsinstitut, Stockholm.

\title{
NOCARDIA ASTEROIDES ALS URSACHE BOVINER MASTITIS
}

\author{
Von \\ K. Hillermark.
}

Bovine Mastitiden, verursacht durch Mikroorganismen der Familie Actinomycetaceae Buchanan, die in der 6. Auflage von Bergey's Manual of Determinative Bacteriology (1948) in die Genera Nocardia und Actinomyces eingeteilt sind, werden in der Literatur relativ selten erwähnt. Im Falle einer Mitteilung solcher liess sich der Infektionsstoff, ohne Einmischung des Begriffes Aktinomykose, in der Regel mit dem Genus Nocardia in Beziehung bringen. Auch in der Veterinärbakteriologie beginnt man immermehr, ähnlich mit dem Verhalten in der Humanbakteriologie, den Aktinomykosebegriff auf solche Fälle zu begrenzen, in denen eine Infektion mit Mikroorganismen vorliegt, welche zum Genus Actinomyces gehören.

Eine in gewissen Teilen Schwedens nicht ungewöhnliche Mastitisform beim Rinde ist die sog. Euteraktinomykose. Diese ist meistens chronischer Art, und das aus den „aktinomykotischen“ Gewebsveränderungen isolierte infektiöse Agens bestand in Reinkulturen des Staphylococcus aureus. Die Bezeichnung Aktinomykose für dieses Euterleiden ist deshalb als unrichtig und irreführend zu betrachten und gründet sich vor allem auf die Struktur der histo-pathologischen Gewebsveränderungen, die im grossen mit derjenigen der echten Aktinomykose übereinstimmen. Mit Rücksicht auf die Ätiologie und den Verlauf ist jedoch dieses Euterleiden eine Staphylokokkenmastitis subakuter bis chronischer Natur. Zur Verhinderung einer Verwirrung in der Nomenklatur wurde deshalb vorgeschlagen, diese Form von Staphylokokkenamstitis als Staphylokokkose (Buer, 1939), Staphylomykose (Kämpe, 1944) oder Aktinokokkose (Tanner, 1952) zu bezeichnen. 
Ob mit Nocardiose pathologische, durch verschiedene Nocardia-Spezies verursachte Zustände gemeint sind, dürfte nicht klar ausgedrückt sein. Gewisse Verfasser, z. B. Larsen et al. (1959), bezeichnen mit Nocardiose "a granulomatous and suppurative infectious disease which is caused by the aerobic actinomycete Nocardia asteroides. Manifestations may be acute or chronic, localized, or generalized".

Höchstwahrscheinlich dürfte Nocardia asteroides diejenige Nocardia-Art sein, welche das grösste Interesse in der Beziehung beansprucht, dass sie sowohl für Menschen als auch Tiere pathogen ist.

Aus dem Gehirnabszess eines Menschen isolierte Eppinger (1891) einen aeroben, säurefesten Mikroorganismus, der nunmehr mit Nocardia asteroides bezeichnet wird. Infektionen mit diesem Aktinomyceten bei Menschen sind danach in einer Reihe von Fällen mitgeteilt worden. Cruz und Clanzy (1952) liefern eine Uebersicht von 40 Fällen, von welchen bei 33 eine Lokalisation allein in den Lungen oder in diesen und anderen Organen gleichzeitig vorlag. Nach 1952 werden mehrere Fälle von Nocardiose erwähnt, und 1959 berichten Larsen et al. über 7 Fälle dieser Krankheit.

Beim Hund sind mehrere Fälle von Nocardiose beschrieben. In einer Reihe von Fällen gründet sich die Diagnose allein auf die histo-pathologischen Gewebsveränderungen, z.B. Moss (1956), in anderen Fällen wiederum wurde ausserdem Nocardia asteroides isoliert - Ginsberg und Little (1948), Cross et al. (1953), Blake (1954), Fey et al. (1954) und Awad (1959). Die krankhaften Veränderungen waren in der Regel auf die Schlundregion, Lungen mit Lymphknoten, Pleura und das Peritonaeum lokalisiert.

Nocardia asteroides wurde in mehreren Fällen auch vom Rind isoliert. Bishop und Fenstermacher (1933) züchteten den Mikroorganismus aus Herden in der Pleura und dem Diaphragma, bei denen zunächst der Verdacht vorlag, sie seien tuberkulöser Natur. Aus Kotyledonen, die von zwei Abortus-Nachgeburten stammten, wurde diese Nocardia-Art von Fey et al. (1954) isoliert.

Ueber den ersten Fall boviner, von Nocardia asteroides verursachter Mastitis wurde aus Australien von Munch-Petersen (1954) berichtet. Dieser Mikroorganismus liess sich aus dem Sekret der angegriffenen Euterviertel während einer Zeit von viereinhalb 
Monaten in Reinkultur isolieren. Experimentell erzeugte MunchPetersen eine fibröse, noduläre Mastitis mit gut abgekapselten Abszessen, die grüngelben Eiter enthielten. Pier et al. (1958) isolierten Nocardia asteroides aus den Eutersekreten von 26 Kühen und 2 Erstlingskalb-Sterken in einem kalifornischen Bestande von 157 Tieren. Der Actinomycet wurde in den Eutersekreten in einem bis drei verschiedenen Fällen in Reinkultur oder in Kombination mit Staphylococcus aureus, Streptococcus agalactiae oder Pseudomonas aeruginosa ermittelt. Ein Tier von den infizierten starb. Eine Erstlingskalb-Sterke, von der Nocardia asteroides in Reinkultur in drei verschiedenen Fällen isoliert worden war, wurde geschlachtet. Das Euter zeigte eine diffuse Fibrose in den dorsalen Gebieten, in den ventralen Partien fanden sich mehrere gut begrenzte Abszesse vom Durchmesser von 2,5-5 cm sowie kleinere über das sekretorische Gewebe verstreute solche. Histo-pathologisch lagen eine extensive Fibrose, gut abgekapselte Granulome mit nekrotischem Zentrum und hypertrophische Veränderungen im Milchgang- und Alveolarepithel vor. Ähnliche Veränderungen wurden bei experimenteller Infektion erzielt. Die für Aktinomykost charakteristischen Keulen- und Rosettenbildungen fehlten. Munch-Petersen hat solche ebenfalls nicht wahrgenommen.

Schliesslich sei erwähnt, dass Dafaalla und Gharib (1958) bei einer Ziege eine durch Nocardia asteroides verursachte Mastitis konstatiert haben.

Durch Nocardia asteroides bewirkte bovine Mastitiden scheinen wenigstens zur Zeit zu den seltener diagnostizierten Mastitisformen zu gehören. In der verfügbaren Literatur vermochte ich nur diejenigen Fälle zu finden, welche von Munch-Petersen (1954) und Pier et al. (1958) mitgeteilt wurden. Um die Kenntnis derartiger Mastitiden und über den diesbezüglichen Mikroorganismus zu bereichern, kann es von Interesse sein, die bei einer in Schweden durch Nocardia asteroides verursachten Mastitis gemachten Erfahrungen vorzulegen.

\section{EIGENE UNTERSUCHUNGEN}

Die klinischen Daten wurden von dem Tierarzt ${ }^{1}$ ) geliefert, dem die tierärztliche Aufsicht des betreffenden Bestandes obliegt; dieser umfasst 25 Kühe und befindet sich im Westen Schwedens.

1) Distriktstierarzt Sture A. Nilsson sei hierfür bestens gedankt. 
Klinische Angaben.

Die 7 Jahre alte Kuh erkrankte am dritten Tage nach dem Kalben an akuter Mastitis (rechtes hinteres Euterviertel) mit hohem Fieber $\left(41^{\circ} \mathrm{C}\right)$ und hoher Pulsfrequenz (96). Das Sekret des angegriffenen Euterviertels war deutlich verändert. Die Kuh wurde mit Dimezathine (Sulfadimidin) intravenös und mit Vaccapenin (Penicillin + Streptomycin) lokal behandelt. Am folgenden Tage waren Euterstatus, Temperatur und Pulsfrequenz unverändert. Eine Sekretprobe wurde zur bakteriologischen Untersuchung entnommen und das Euterviertel mit Aureomycin behandelt. Eine Allgemeinbehandlung mit Dimezathine - $70 \mathrm{~g}$ tgl. per os — fand an diesem Tage und am folgende Tage statt. Da sich am vierten Krankheitstage keine Besserung wahrnehmen liess, wurde die Sulfadimidinbehandlung gegen eine intramuskuläre Penicillinbehandlung und eine Lokalbehandlung mit Terramycin ausgetauscht, nachdem eine zweite Sekretprobe zur bakteriologischen Untersuchung entnommen worden war. Zu diesem Zeitpunkt waren die Temperatur und der Appetit der Kuh herabgesetzt. Die Euterentzündung wie auch das Allgemeinbefinden (Temp. $40^{\circ}-41^{\circ} \mathrm{C}$, Pulsfrequenz 92-100) wurden von den Antibiotikabehandlungen wenig beeinflusst, weshalb das betreffende Euterviertel am zwölften Krankheitstage ausser Funktion gesetzt wurde. Nach dieser Massnahme besserten sich das Allgemeinbefinden und der Appetit, wenngleich sich die Temperatur fortgesetzt hoch, um $40^{\circ} \mathrm{C}$ hielt. Eine Allgemeinbehandlung mit grossen Dosen Terramycin wurde deshalb am 15. und 17. Krankheitstage vorgenommen, ohne dass das Fieber dadurch beeinflusst wurde. Dreiundzwanzig Tage nach Feststellung der Mastitis wurde ein im angegriffenen Euterviertel ventral gelegener, von einer dicken Kapsel umgebener Abszess auf chirurgischem Wege geöffnet. Der Abszessinhalt wurde einer bakteriologischen Untersuchung unterzogen. - Nach diesem chirurgischen Eingriff besserte sich das Allgemeinbefinden und die Temperatur fiel auf $39^{\circ} \mathrm{C}$. Sobald die Kuh fieberfrei geworden war, wurde sie zur Schlachtung gesandt.

Leider wurde das Euter bei der Schlachtung nicht zur pathologisch-anatomischen Untersuchung aufgehoben. Es wurde jedoch festgestellt, dass das angegriffene Euterviertel hochgradig vergrössert war und den Sitz einer ausgeprägten Fibrose gebildet hatte.

\section{UNTERSUCHUNG DER SEKRETPROBEN UND DES ABSZESS- INHALTS}

Beide Sekretproben hatten grauliche Farbe und enthielten gelbgraue Flocken. Der „California mastitis test“ (CMT) nach Schalm und Noorlander (1957) war positiv bezw. stark positiv. Die Leukozytenmenge pro $\mathrm{ml}$ Sekret betrug zirka 650,000 bezw. 2 Millionen. Die Sedimente hatten graugelbe Farbe und beliefen sich auf 4 bezw. 10 Vol.\%. Die mikroskopische Untersuchung der Sedimente zeigte in beiden Fällen nur lange, grampositive, verzweigte und nichtverzweigte sowie unregelmässig gefärbte Fila- 
mente. Diese waren bei der Färbung nach Ziehl-Neelsen und Entfärbung mit $3 \% \mathrm{HCl}$ enthaltendem Salzsäurespiritus nicht säurefest.

Der Abszessinhalt war viskos, gelbgrau und enthielt gelbgrüne Klumpen. Ausserdem kamen in demselben zahlreiche kleine weisse Partikeln vor. Diese waren weich und bestanden mikroskopisch aus dichtliegenden Filamenten (Mycelium). Grampositive Filamente, die morphologisch mit den in den Sekretproben wahrgenommenen übereinstimmten, fanden sich auch im Abszessinhalt. Dieselben waren bei der oben angegebenen Färbemethode nicht säurefest.

\section{ISOLIERUNG DES MIKROORGANISMUS}

Kulturversuche auf Rinderblutagar ( $7 \%$ Blut) mit den beiden Sekretproben ergaben bei $37^{\circ} \mathrm{C}$ kein Wachstum, und zwar weder unter aeroben noch anaeroben Verhältnissen.

Die Primärkultur auf derselben Art von Agar aus dem Abszessinhalt zeigte nach 24 Stunden langer Inkubation bei $37^{\circ} \mathrm{C}$ unter aeroben Verhältnissen äusserst kleine, weisse Kolonien, die unter dem Mikroskop den Eindruck daunenartiger bis wolliger Formationen machten. Nach 48 Stunden langer Inkubation wurde eine Reinkultur von kleinen, zirka $1 \mathrm{~mm}$ im Diameter messenden, weissen, leicht erhabenen Kolonien beobachtet. Diese Kolonien hatten in diesem Stadium eine gewisse Ähnlichkeit mit

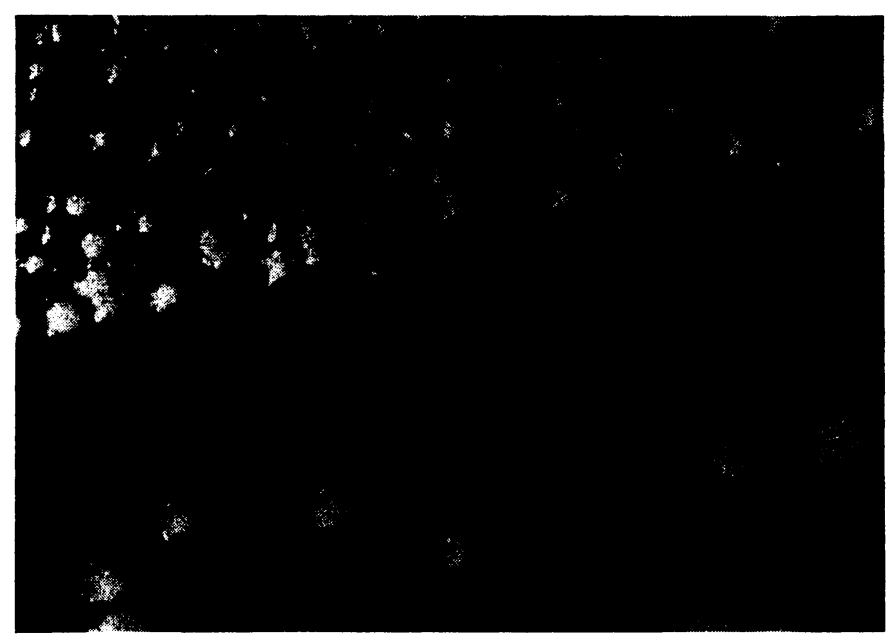

A b b i 1 d. 1. Oberflächenkolonien auf Rinderblutagar nach 96 Stunden langer Inkubation (Primärkultur). $10 \times$. 
Staphylokokken oder Mikrokokken. Nach 96 Stunden währender Inkubation waren die Kolonien etwas grösser, opak, deutlich konvex und hatten leicht granulierte Flächen sowie halbdurchsichtige, radiär streifige Ränder (Abbild. 1). Die Kolonien waren nunmehr gleichsam ins Substrat herabgesenkt und hafteten an diesem, ferner hatten sie eine hellgelbe Farbe anzunehmen begonnen, die sich bei fortgesetzter Inkubation verstärkte. Die Pigmentierung war auf die konvexen zentralen Teile der Kolonien lokalisiert, während die peripheren radiär streifigen Randzonen weiss aussahen. Ein Luftmycelium liess sich nicht wahrnehmen. - Bei anaerober Inkubation auf Rinderblutagar wurde kein Wachstum bei $37^{\circ} \mathrm{C}$ erhalten.

\section{SPEZIELLE EIGENSCHAFTEN DES ISOLIERTEN MIKRO- ORGANISMUS}

Agar mit $7 \%$ Rinderblut. Nach 48-72 Stunden langer Inkubation (Subkultur) bei $37^{\circ} \mathrm{C}$ begannen die relativ weit voneinander liegenden Oberflächenkolonien ein Aussehen anzunehmen, das sich von den dichter wachsenden Kolonien unterschied. Ausser ihrem grösseren Umfang hatte ein kleiner Prozentsatz derselben Sternform angenommen, während andere ein in Abbildung 2 veranschaulichtes Aussehen zeigten oder die Bildung einer kleinen Warze zentral auf konvexen Kolonien aufwiesen. In den Gebieten mit dichtem Wachstum behielten die Kolonien

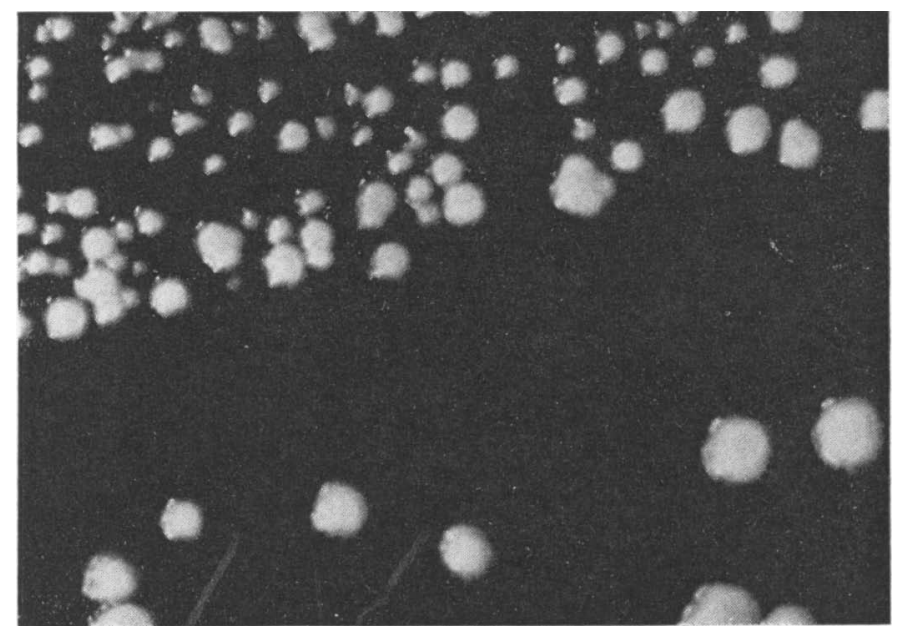

A b b i ld. 2. Oberflächenkolonie auf Rinderblutagar nach 96 Stunden langer Inkubation (Subkultur). $50 \times$. 
der konvexen Form ihr früher beschriebenes Aussehen (Abbild. 1) bei. Subkulturen von sternförmigen Kolonien, von Kolonien des Typs in Abbildung 2 oder von solchen konvexen mit Warzen ergaben konvex verbleibende Kolonien mit relativ dichtem Wachstum, während Kolonien mit grösserem Lebensraum in die Sternform oder andere oben beschriebene Formen übergingen. Sämtliche Kolonietypen bildeten ein gelb-orangegelb, schwach lachsfarbenes Pigment. Die intensivste Pigmentbildung wurde dann erhalten, wenn die Plattenkulturen nach 4-5 Tage langer Aufbewahrung im Thermostaten bei Zimmertemperatur unter Einwirkung des Tageslichts augestellt wurden. Nach 9-10 Tage langer Inkubation wurde schwache Hämolyse wahrgenommen; eine solche trat nicht ein, wenn das Rinderblut gegen Pferdeblut ausgetauscht wurde.

Subkulturen auf Agar mit Rinderblut oder Pferdeblut zeigten nach 4 Tage langer Inkubation bei $37^{\circ} \mathrm{C}$ keine Hämolyse. Kulturen auf Rinderblut- und Pferdeblutagar wurden danach teils im Kühlschrank $\left(+4^{\circ} \mathrm{C}\right)$ und teils bei Zimmertemperatur aufbewahrt. Im ersteren Falle war in den Rinderblutplatten nach 4 Tagen totale Hämolyse eingetreten, jedoch nicht ringsum die Kolonien. Diese waren von einer scharfen roten Zone intakter Blutkörperchen umgeben. Dieses Phänomen trat nicht in Plattenkulturen auf, die Pferdeblut enthielten, und zwar weder bei ihrer Aufbewahrung im Kühlschrank noch bei Zimmertemperatur. Die Rinderblut enthaltenden Plattenkulturen, welche bei Zimmertemperatur aufbewahrt wurden, zeigten nach 4 Tagen geringgradige Hämolyse, die aber an Klarheit und Deutlichkeit mit der Hämolyse der im Kühlschrank gehaltenen Plattenkulturen nicht zu vergleichen war.

Glykoseagar. Die Kolonien waren hier in der Regel grösser als auf Blutagar, nicht so konvex wie auf diesem, sondern unregelmässiger und trocken mit orange-lachsfarbenem Zentrum und weisser radiärer Randzone.

Löwensteins Substrat mit 0,75\% und $6 \%$ Glyzerin. Dieses Substrat ergab gutes Wachstum von Kolonien, die in gewissem Grade an humane Tuberkelbazillenkolonien erinnerten.

Gelatinestich. Im Stich selbst war kein Wachstum vorhanden. Das Oberflächenwachstum kennzeichnete sich durch leicht erhabene, anfangs weisse Kolonien, die später bei einem Durchmesser von zirka 3-4 mm einen opaken, gefaltenen, orangegefärbten zentralen Teil und eine weisse halbdurchsichtige Randzone auf- 
wiesen. Eine Verflüssigung der Gelatine war selbst nach 60 Tagen nicht eingetreten, und zwar weder bei $22^{\circ}$ noch bei $37^{\circ} \mathrm{C}$.

Serumbouillon. Nach 18 Stunden langer Inkubation bei $37^{\circ} \mathrm{C}$ hatten sich eine sehr dünne, weisse Oberflächenhaut und ein unbedeutender daunenartiger Bodensatz gebildet. Bei fortgesetzter Inkubation nahm der Bodensatz zu und die Haut wurde dicker, wobei auf derselben gelbe bis orangegefärbte, scheibenartige Gebilde auftraten. Diese und Teile der Oberflächenhaut sonderten sich allmählich vom übrigen Teil derselben und sanken zu Boden; der zwischen Haut und Sediment vorhandene Teil der Bouillon verblieb jedoch stets klar.

Methylenblaumilch. Eine Reduktion trat innerhalb von 24 Stunden in den Konzentrationen $1: 20.000,1: 10.000$ und 1:5.000 ein. Nach 2 Tagen kehrte die blaue Farbe wieder, die danach 28 Tage lang bestehen blieb. Keine Koagulation.

Lackmusmilch ergab langsame Alkalinisierung. Keine Koagulation in 30 Tagen.

Morphologie und Färbungseigenschaften. 96 Stunden gewachsene Blutagarkulturen (Primär- und Subkulturen) zeigten überwiegend grampositive, stäbchenförmige und kokkoide Elemente sowie sparsamer grampositive, kurze, verzweigte Filamente. Bei Färbung nach Ziehl-Neelsen und Entfärbung mit $3 \% \mathrm{HCl}$ enthaltendem Salzsäurespiritus wiesen vor allem die kokkoiden Formen Säurefestigkeit auf.

In 18 Stunden alter Serumbouillonkultur fanden sich fast nur längere und kürzere, grampositive Filamente, von denen manche stark verzweigt waren, andere dagegen keine Verzweigung oder den Beginn von Verzweigung (Knospung) zeigten. Eine Reihe von Filamenten oder Segmenten solcher waren bei der Färbung nach Ziehl-Neelsen und der vorher genannten Entfärbung schwach säurefest. Nach 72 Stunden langer Inkubation dominierten grampositive, stäbchenförmige und kokkoide Elemente; die Filamente waren nun kürzer und bedeutend weniger verzweigt als nach 18 Stunden dauernder Inkubation. Eine geringere Anzahl von Filamenten oder Segmenten solcher war schwach säurefest. Die stäbchenförmigen und kokkoiden Elemente kennzeichneten sich durch Säurefestigkeit in grösserem Umfange als die Filamente, auch im Grade der Säurefestigkeit übertrafen sie die. Filamente. Nach fortgesetzter Inkubation schien die Säurefestigkeit bei den Stäbchen und den kokkoiden Formen zuzunehmen.

Fermentative Eigenschaften. In einer Mischung von Pepton- 
wasser mit Fleischextraktbouillon (zuckerfrei!) mit oder ohne Serum und Zusatz nachstehend angegebener Zuckerarten u.a. für sich trat bei 30 Tage langer Inkubation bei $37^{\circ} \mathrm{C}$ weder Säure noch Gas auf. Die geprüften Kohlenhydrate, Alkohole bezw. Glykoside waren folgende: Arabinose, Rhamnose, Xylose, Dextrose, Fruktose, Galaktose, Mannose, Laktose, Maltose, Saccharose, Trehalose, Raffinose, Stärke, Dextrin, Glykogen, Inulin, Adonit, Dulcit, Mannit, Sorbit, Inosit, Salicin, Amygdalin und Arbutin. NaHippurat wurde in 30 Tagen nicht hydrolysiert. Aesculin wurde nach 6 Tagen hydrolysiert, doch ohne gleichzeitige Säurebildung. Die ursprünglich bläulich fluoreszierende Farbe war noch vorhanden, Zusatz von Ferrizitratlösung (1\%) gab jedoch eine intensisv schwarze Farbe.

Biochemische Eigenschaften. Indol wurde nicht gebildet. Die Methylrot- und die Voges-Proskauer-Reaktion waren negativ. Natriumzitrat und Paraffin liessen sich als Kohlenstoffquellen ausnutzen. Harnstoff wurde zerlegt. Schwefelwasserstoff wurde in geringer Menge gebildet, und Nitrat zu Nitrit reduziert.

Thermische Eigenschaften. 24 und 48 Stunden alte Serumbouillonkulturen wurden im Wasserbad durch 30 Minuten lange Einwirkung von Temperaturen von $64^{\circ}, 66^{\circ}, 68^{\circ}$ und $70^{\circ} \mathrm{C}$ in ihrer Lebensfähigkeit nicht beeinträchtigt; dasselbe gilt für Temperaturen von $72^{\circ}, 74^{\circ}$ und $76^{\circ} \mathrm{C}$ und die Zeit von 30 Sekunden.

Pathogenität. Meerschweinchen, die mit $0,7 \mathrm{ml} 48$ Stunden alter Serumbouillonkultur intraperitoneal geimpft wurden, starben nach 5 bis 9 Tagen. Das parietale und das viscerale Peritonäum waren bei der Sektion von $1 / 2-1 \mathrm{~mm}$ grossen, weissen, etwas erhabenen Neubildungen gleichsam besät, die in geringerer Anzahl auch auf der Pleura diaphragmatica, pulmonalis und costalis vorkamen. Zwischen den Leberlappen, zwischen Leber und Diaphragma sowie zwischen der Leber und dem parietalen Peritonäum fanden sich ausgeprägte Adhärensen. Die histopathologische Untersuchung ergab subakute fibrinopurulente Peritonitis (Bauchwand, Leber und Milz), miliare zellige Lebernekrosen, gelinde zentrolobuläre einfache Leberzellenverfettung sowie RE-Proliferation in der roten Milzpulpa.

Die mikroskopische Untersuchung der Neubildungen zeigte grampositive, verzweigte und nichtverzweigte Filamente, die mit den in Eutersekretproben und im Abszessinhalt ermittelten Filamenten übereinstimmten. Die Filamente erwiesen sich beim Ge- 
brauch der vorher erwähnten Färbungs- und Entfärbungsmethodik als partiell säurefest.

Der betreffende Mikroorganismus wurde aus dem Herzblut, den Peritonealläsionen, der Bauch- und Brusthöhlenflüssigkeit, dem Leber- und Lungenparenchym sowie aus der Milzpulpa auf Rinderblutagar isoliert.

Die intraperitoneale Impfung mit $0,7 \mathrm{ml}$ Filtrat aus einer 48 Stunden alten Serumbouillonkultur beeinflusste Meerschweinchen nicht. Die Obduktion der nach 4 Wochen getöteten Tiere ergab keine makroskopisch wahrnehmbaren Gewebeveränderungen.

Die Todesursache bei den mit Kultur geimpften Tieren kann möglicherweise auf einem Zusammenwirken zwischen dem Mikroorganismus und einer mit diesem verbundenen toxischen Substanz beruht haben.

Mäuse waren gegen die intraperitoneale Inokulierung des Mikroorganismus resistent. Das Sektionsbild dieser vier Wochen später getöteten Tiere war ganz negativ.

Empfindlichkeit für Chemotherapeutika und Antibiotika. Die Bestimmung derselben geschah nach der sog. Papierscheibenmethode. Die technische Durchführung und die Deutung der Resultate (Tab. 1) erfolgte nach den zur Zeit in Schweden gebräuchlichen Normen, Ericsson et al. (1954 und 1959).

Tabelle 1.

Empfindlichkeitsbestimmungen in vitro.

\begin{tabular}{l|c|l}
\hline & $\begin{array}{c}\text { Minimikonz. } \\
\text { für tolale Hemmung }\end{array}$ & $\begin{array}{l}\text { Grad der Empfindlichkeit } \\
\text { (klinische Deutung) }\end{array}$ \\
\hline \begin{tabular}{l|l} 
Sulfaisodimidin \\
Nitrofurantoin
\end{tabular} & $\begin{array}{r}0,5 \mathrm{mg} \% \\
\text { Penicillin }\end{array}$ & $\begin{array}{l}\text { empfindlich (partiell) } \\
\text { resistent }\end{array}$ \\
Erythromycin & $12,5 \mathrm{mg} \%$ & resistent \\
Streptomycin & $64 \mathrm{mcg} / \mathrm{ml}$ & resistent \\
Chlortetracyklin & $5 \mathrm{mcg} / \mathrm{ml}$ & ziemlich empfindlich \\
Oxytetracyklin & $250 \mathrm{mcg} / \mathrm{ml}$ & resistent \\
Tetracyklin & $50 \mathrm{mcg} / \mathrm{ml}$ & wenig empfindlich \\
Chloramphenicol & $25 \mathrm{mcg} / \mathrm{ml}$ & wenig empfindlich \\
Bacitracin & $64 \mathrm{mcg} / \mathrm{ml}$ & resistent \\
Neomycin & $>16 \mathrm{I.U} / \mathrm{ml}$ & resistent \\
Novobiocin & $0,05 \mathrm{mcg} / \mathrm{ml}$ & ziemlich empfindlich \\
Oleandomycin & $>300 \mathrm{mcg} / \mathrm{ml}$ & resistent \\
Polymyxin & $>50 \mathrm{mcg} / \mathrm{ml}$ & resistent \\
& - & resistent
\end{tabular}




\section{DISKUSSION}

Das klinische Bild, vor allem der Charakter des Eutersekrets, sprechen unzweideutig für eine Mastitis. Die ausgebreitete Fibrose und die Abszessbildung im ventralen Teil des Euterviertels stimmen mit den früher bei Euternocardiose gemachten Beobachtungen überein. Verzweigte und nichtverzweigte grampositive Filamente wurden in Ausstrichpräparaten aus dem Sediment des Eutersekrets in zwei verschiedenen Fällen und im Abszessinhalt in einem dritten Fall wahrgenommen. Nur im letztgenannten Fall gelang es, den Mikroorganismus in Kultur zu erhalten. Die Erklärung dafür, dass eine Isolierung dieses Mikroorganismus in früheren Untersuchungen weniger erfolgreich war, kann in den vor den Probenentnahmen angewandten, verhältnismässig intensiven Antibiotikabehandlungen und in der partiellen Empfindlichkeit des Mikroorganismus für Sulfapräparate gelegen haben.

Mit Rücksicht auf die morphologischen, biologischen, biochemischen und pathogenen Eigenschaften des isolierten Mikroorganismus stimmt derselbe im grossen ganzen mit der von Munch-Petersen (1954) und Pier et al. (1958) aus Mastitiseutern isolierten Nocardia asteroides überein. Gewisse Unterschiede lassen sich jedoch feststellen. So besassen wenigstens Subkulturen auf Rinderblutagar keine einheitlichen Kolonieformen, sondern diese variierten von konvexen, granulierten zu sternförmigen, kegelförmigen und anderen Kolonietypen in derselben Plattenkultur. Der Stamm hydrolysierte Aesculin ohne gleichzeitige Säurebildung und kennzeichnete sich durch stärkere Wärmeresistenz sowie andere Empfindlichkeit gegen Antibiotika in vitro als der von Pier et al. isolierte Stamm.

Trotz der angeführten Unterschiede dürften die angeführten morphologischen, biologischen, fermentativen, biochemischen und pathogenen Eigenschaften des gefundenen Mikroorganismus dazu berechtigen, ihn mit Nocardia asteroides zu identifizieren. Die Diagnose wurde später auch von einem anderen bakteriologischen Laboratorium ${ }^{2}$ ) bestätigt.

Die biologischen Verschiedenheiten, die zwischen den wenigen bisher in verschiedenen Ländern der Erde aus Mastitiseutern iso-

2) Für die freundliche Uebernahme der diesbezüglichen Untersuchung dankt der Verfasser besten Herrn Dr. H. Paldrok, Statens Bakteriologiska Laboratorium, Stockholm. 
lierten Nocardia asteriodes-Stämmen vorzuliegen scheinen, und ferner nicht zum wenigsten ihre verschiedene Empfindlichkeit für die gleichen Antibiotika in vitro könnten möglicherweise auf mikrobiologische Varianten hindeuten.

Durch Nocardia asteroides verursachte Mastitiden dürfen vom Gesichtspunkte der Volksgesundheit nicht ausser acht gelassen werden. Pasteurisierung inaktiviert nicht immer den Mikroorganismus. Der von mir isolierte Stamm überlebte gut die Temperatur und Zeit, die in Schweden bei der Pasteurisierung angewandt werden ( $74^{\circ} \mathrm{C} 15$ Sekunden lang).

\section{LITERATUR}

1. Awad, F. I.: Zbl. Vet. Med., 1959, 6, 919.

2. Bergey's Manual of Determinative Bacteriology: 6th Ed., Williams and Wilkins Co., Baltimore, 1948.

3. Bishop, L. M. and Fenstermacher, R.: Cornell Vet. 1933, 23, 288.

4. Blake, W. P.: J. Amer. vet. med. Ass. 1954, 125, 467.

5. Buer, A. W.: Skand. VetTidskr. 1939, 29, 13.

6. Cross, R. F., Nago, W. T. and Morrison, R. H.: J. Amer. vet. med. Ass. 1953, 123, 535.

7. Cruz, P. T. and Clanzy, C. F.: Amer. J. Path. 1952, 28, 607.

8. Dafaalla, E. N. and Gharib, H. M.: Brit. Vet. J., 1958, 114, 143.

9. Eppinger, H.: Beitr. path. Anat. 1891, 9, 289.

10. Ericsson, H., Högman, C. and Wickman, K.: Scand. J. clin. Lab. Invest., 6. suppl., 1954, 11, 21.

11. Ericsson, H. and Swartz-Malmberg, G.: Antibiotica et Chemotherapia, Karger, Basel/New York 1959, 6, 41.

12. Fey, H., Holm, P. und Teucher, E.: Schweiz. Arch. Tierheilk. 1954, $96,642$.

13. Ginsberg, A. and Little, A. C. W.: J. Path. Bact. 1948, 60, 563.

14. Kämpe, A.: Skand. VetTidskr. 1944, 34, 65.

15. Larsen, M. C., Diamond, H. D. and Collins, H. S.: Arch. intern. Med., 1959, 103, 712.

16. Moss, L. C.: J. Amer. vet. med. Ass. 1956, 128, 143.

17. Munch-Petersen, E.: Aust. vet. J., 1954, 30, 297.

18. Pier, A. C., Gray, D. M. and Fosatti, M. J.: Amer. J. Vet. Res. 1958, $19,319$.

19. Schalm, O. W. and Noorlander, D. O.: J. Amer. vet. med. Ass. 1957, 130, 199.

20. Tanner, A.: Nord. Vet.-Med. 1952, 4, 655.

\section{ZUSAMMENFASSUNG}

Verf. beschreibt klinisch eine bovine Mastitis, die mit Fiebertemperaturen bis $\mathrm{zu} 41^{\circ} \mathrm{C}$ verlief und zur Entwicklung ausgebreiteter Fibrose nebst Abszessbildung im ventralen Teil des angegriffenen Euterviertels führte. 
Nur verzweigte und nichtverzweigte, grampositive Filamente wurden im graulichen, gelbgraue Flocken enthaltenden Sekret und im viskosen, gelbgrauen, gelbgrüne Flocken und weisse Myceliumklumpen enthaltenden Abszessinhalt wahrgenommen.

Die morphologischen, kulturellen, fermentativen, biochemischen und pathogenen Eigenschaften des Mikroorganismus, die beschrieben werden, berechtigen nach Auffassung des Verfassers dazu, den isolierten Mikroorganismus mit Nocardia asteroides zu identifizieren.

\section{SUMMARY}

Nocardia asteroides as a cause of bovine mastitis.

A clinical description is given of a form of bovine mastitis accompanied by fever up to $41^{\circ} \mathrm{G}$ and leading to diffuse fibrosis with abscessformation in the ventral portion of the affected quarters.

The mammary secretion was grey with yellowish-grey clumps. Abscess contents were viscous, yellowish-grey, and mixed with yellowish-green solid material and white mycelial clumps. Branching and non-branching Gram-positive filaments were the only organisms seen in smears.

According to the author's opinion the morphological, cultural, fermentative, biochemical and pathogenic properties of the organism isolated justify its identification as Nocardia asteroides.

\section{SAMMANFATTNING}

Nocardia asteroides som orsak till bovin mastit.

Författaren lämnar en klinisk beskrivning av en bovin mastit förlöpande med temperatur upp till $41^{\circ} \mathrm{C}$ och utvecklande sig till utbredd fibros samt abscessbildning $i$ den angripna juverdelens ventrala del.

Endast förgrenade och icke förgrenade grampositiva filament iakttogos i det förändrade sekretet, gråaktigt med gulgrå flockor, samt i det viskösa, gulgrå abscessinnehållet med gulgröna flockor och vita mycelieklumpar.

Den isolerade mikroorganismens morfologiska, kulturella, fermentativa, biokemiska och patogena egenskaper, vilka beskrivas, anses berättiga dess identifiering som Nocardia asteroides.

(Received May 19. 1960). 